Homology, Homotopy and Applications, vol.21(2), 2019, pp.23-26

\title{
SPACES WITH COMPLEXITY ONE
}

\author{
ALYSON BITTNER
}

\author{
(communicated by John R. Klein)
}

\begin{abstract}
An $A$-cellular space is a space built from a space $A$ and its suspensions, analogous to the way that $C W$-complexes are built from $S^{0}$ and its suspensions. The $A$-cellular approximation of a space $X$ is an $A$-cellular space $C W_{A} X$, which is closest to $X$ among all $A$-cellular spaces. The $A$-complexity of a space $X$ is an ordinal number that quantifies how difficult it is to build an $A$-cellular approximation of $X$. In this paper, we study spaces with low complexity. In particular, we show that if $A$ is a sphere localized at a set of primes then the $A$-complexity of each space $X$ is at most 1 .
\end{abstract}

\section{Introduction}

Let $A$ be a pointed $\mathrm{CW}$-complex. An $A$-cellular space is a space built out of copies of $A$ via iterated pointed homotopy colimit constructions. Given a pointed space $X$, the $A$-cellular approximation of $X$ is an $A$-cellular space $C W_{A} X$ equipped with a map $C W_{A} X \rightarrow X$, such that the induced map of pointed mapping complexes $\operatorname{Map}_{*}\left(A, C W_{A} X\right) \rightarrow \operatorname{Map}_{*}(A, X)$ is a weak equivalence. For example, $S^{0}$-cellular spaces are CW-complexes and an $S^{0}$-cellular approximation of a space $X$ is the $\mathrm{CW}$-approximation of $X$. Farjoun [2] showed that the $A$-cellular approximation of $X$ exists for any $A$ and $X$ and that it is unique up to homotopy equivalence. The space $C W_{A} X$ is the best possible approximation of $X$ in the class of $A$-cellular spaces: for any $A$-cellular space $Y$ and a map $Y \rightarrow X$ there exists a map $Y \rightarrow C W_{A} X$, unique up to homotopy, such that the following diagram is homotopy commutative.

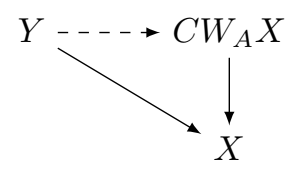

In [1] Chachólski, Dwyer, and Intermont introduced the concept of the $A$-complexity of a space $X$, which is the minimum ordinal number of homotopy colimits necessary to produce $C W_{A}(X)$ from copies of $A$. More precisely, starting with $\mathbf{C}_{0}$ the full subcategory of pointed spaces such that objects of $\mathbf{C}_{0}$ have the homotopy type of a retract of wedges of $A$, one can construct an increasing chain of categories indexed

Received February 5, 2018, revised September 6, 2018; published on December 19, 2018. 2010 Mathematics Subject Classification: 55P60, $18 \mathrm{C} 10$.

Key words and phrases: cellular space, complexity, mapping space.

Article available at http://dx.doi.org/10.4310/HHA.2019.v21.n2.a2

Copyright (C) 2018, Alyson Bittner. Permission to copy for private use granted. 
by ordinal numbers $\alpha$, where $\mathbf{C}_{\alpha}$ is the category of all spaces of the homotopy type of hocolim $\mathbf{D} F$ for some small category $\mathbf{D}$ and a functor $F: \mathbf{D} \rightarrow \bigcup_{\beta<\alpha} \mathbf{C}_{\beta}$ and their retracts. The $A$-complexity of $X$ is the minimal ordinal $\kappa_{A}(X)$ such that $X \in \mathbf{C}_{\kappa_{A}(X)}$.

A CW-approximation of a space $X$ can be constructed as $|\operatorname{sing} X|$, which is a homotopy colimit of discrete spaces. Thus, in the case of $A=S^{0}$ the category $\mathbf{C}_{1}$ consists of all spaces having the homotopy type of a CW-complex, which implies that $\kappa_{S^{0}}(X) \leqslant 1$ for any space $X$. This is, however, not the case for other choices of $A$, and in general the complexity of a space need not even be finite. For example, if $A=$ $M(\mathbb{Z} / p, n)$ is a Moore space for some $n \geqslant 1$ with $p$ a prime and $X=M\left(\mathbb{Z} / p^{\infty}, n+1\right)$ then $\kappa_{A}(X)=\omega$ (see [1, Proposition 9.3]).

Results of Stover [7] imply that if $A=S^{n}$ then $\kappa_{A}(X) \leqslant 2$ for all $X$. Chachólski, Dwyer and Intermont observed for $A=S^{1}$ we have $\kappa_{A}(X) \leqslant 1$ for all $X$ and suggested that it should be possible to similarly lower the bound for all spheres $A=S^{n}[1, \S 9.3]$. The goal of this note is to show that this is indeed the case, and that this result holds in even greater generality:

Theorem 1.1. If $A$ is a sphere or a sphere localized at a set of primes then $\kappa_{A}(X) \leqslant 1$ for all spaces $X$.

\section{Acknowledgments}

I want to express my appreciation to my advisor, Bernard Badzioch, for his helpful comments which led to the final version of paper. Additionally, I would like to thank Matthew Sartwell for several useful conversations.

\section{Algebras of mapping spaces}

Our proof of Theorem 1.1 will be based on the following algebraic description of mapping spaces obtained by Sartwell [5]. For pointed spaces $X, Y$ let $\operatorname{Map}_{*}^{\Delta}(X, Y)$ denote the simplicial mapping complex of pointed maps $X \rightarrow Y$. Given a pointed CW-complex $A$ let $\mathbf{T}^{A}$ denote a simplicial category on objects $T_{n}$ for $n=0,1, \ldots$, and such that

$$
\operatorname{Hom}_{\mathbf{T}^{A}}\left(T_{n}, T_{m}\right)=\operatorname{Map}_{*}^{\Delta}\left(\bigvee^{m} A, \bigvee^{n} A\right) .
$$

Notice that in this category $T_{n}$ is the $n$-fold product of $T_{1}$. A $\mathbf{T}^{A}$-algebra is a product preserving simplicial functor $\mathbf{T}^{A} \rightarrow \mathbf{\text { sSet }}$ * where $\mathbf{s S e t}_{*}$ is the category of pointed simplicial sets. Let $\mathbf{A l g}^{\mathbf{T}^{A}}$ denote the category of all $\mathbf{T}^{A}$-algebras with natural transformations as morphisms.

Notice that any pointed space $X$ defines a $\mathbf{T}^{A}$-algebra $\Omega^{A}(X)$ such that

$$
\Omega^{A}(X)\left(T_{n}\right)=\operatorname{Map}_{*}^{\Delta}\left(\bigvee^{n} A, X\right) .
$$

The resulting functor $\Omega^{A}: \mathbf{T o p}_{*} \rightarrow \mathbf{A l g}^{\mathbf{T}^{A}}$ has a left adjoint $B^{A}$.

The category $\mathbf{A l g}^{\mathbf{T}^{A}}$ can be equipped with a model category structure where weak equivalences and fibrations are defined as objectwise weak homotopy equivalence 
and Serre fibrations respectively. Denote also by $\mathbf{R}^{A} \mathbf{T o p}_{*}$ the category $\mathbf{T o p}_{*}$ taken with the model category structure where fibrations are Serre fibrations and weak equivalences are maps $f: X \rightarrow Y$ that induce weak equivalences $f_{*}: \operatorname{Map}_{*}^{\Delta}(A, X) \rightarrow$ $\operatorname{Map}_{*}^{\Delta}(A, Y)$. In other words, $\mathbf{R}^{A} \mathbf{T o p}_{*}$ is obtained by taking the right Bousfield localization of the usual model category structure on $\mathbf{T o p}_{*}$ with respect to the space $A[3,5.1 .1]$. Sartwell showed that the following holds:

Theorem 2.1 ([5]). Let $A$ be a sphere or a sphere localized at a set of primes. The adjoint pair

$$
B^{A}: \mathbf{A l g}^{\mathbf{T}^{A}} \rightleftarrows \mathbf{R}^{A} \mathbf{T o p}_{*}: \Omega^{A}
$$

is a Quillen equivalence.

A simplicial version of this argument can be seen in [4].

\section{Proof of Theorem 1.1}

Directly from the definition of an $A$-cellular approximation it follows that if spaces $X$ and $Y$ are weakly equivalent in $R^{A}$ Top then $C W_{A} X$ and $C W_{A} Y$ are weakly homotopy equivalent, and so $\kappa_{A}(X)=\kappa_{A}(Y)$. Let $A$ be a space as in Theorem 1.1. By Theorem 2.1 for any space $X$ we get a weak equivalence $X \simeq B^{A} Q \Omega^{A} X$ in $R^{A}$ Top, where $Q \Omega^{A} X$ denotes a cofibrant replacement of $\Omega^{A} X$ in the category $\mathbf{A l g}^{\mathbf{T}^{A}}$. Therefore, it is only necessary to show that for any space $X, \kappa_{A}\left(B^{A} Q \Omega^{A} X\right) \leqslant 1$.

The algebra $Q \Omega^{A} X$ can be described more explicitly as follows. Let $U: \mathbf{A l g}^{\mathbf{T}^{A}} \rightarrow$ sSet $_{*}$ denote the forgetful functor, $U(\Phi)=\Phi\left(T_{1}\right)$. This functor has a left adjoint $F: \mathbf{s S e t}_{*} \rightarrow \operatorname{Alg}^{\mathbf{T}^{A}}[\mathbf{6}, 2.3]$. For a $\mathbf{T}^{A}$-algebra $\Phi$, let $F U_{\bullet} \Phi$ be the simplicial $\mathbf{T}^{A_{-}}$ algebra defined by the adjoint pair $(F, U)$. Explicitly:

$$
F U_{n} \Phi:=(F U)^{n+1} \Phi .
$$

By $[5,2.3 .3]$ the natural map $\left|F U_{\bullet} \Phi\right| \rightarrow \Phi$ is a cofibrant replacement of $\Phi$ in the category $\mathbf{A} \lg ^{\mathbf{T}^{A}}$.

In view of this fact we need to show that for any space $X$ the $A$-complexity of $B^{A}\left|F U_{\bullet} \Omega^{A} X\right|$ is at most one. The functor $B^{A}$ is a left adjoint and so commutes with homotopy colimits, in particular, with the geometric realization functor. The functor $B^{A} F: \mathbf{s S e t}_{*} \rightarrow \mathbf{T o p}_{*}$ is left adjoint to $U \Omega^{A}$, and since $U \Omega^{A}(X)=\operatorname{Map}_{*}^{\Delta}(A, X)$, thus for $Y$ a (pointed) simplicial set we get $B^{A} F(Y)=A \wedge|Y|$. Combining these observations we obtain

$$
\begin{aligned}
B^{A}\left|F U \cdot \Omega^{A} X\right| \simeq\left|B^{A} F U \cdot \Omega^{A} X\right| & \simeq \operatorname{hocolim}_{n \in \Delta^{o p}}\left|\left(B^{A} F\right) U\left((F U)^{n} \Omega^{A} X\right)\right| \\
& \simeq \operatorname{hocolim}_{n \in \Delta^{o p}}\left|\left(B^{A} F\right)(F U)^{n} \Omega^{A} X\left(T_{1}\right)\right| \\
& \simeq \operatorname{hocolim}_{n \in \Delta^{o p}} A \wedge\left|(F U)^{n} \Omega^{A} X\left(T_{1}\right)\right| \\
& \simeq \operatorname{hocolim}_{(n, m) \in \Delta^{o p} \times \Delta^{o p}} A \wedge\left((F U)^{n} \Omega^{A} X\left(T_{1}\right)\right)_{m} \\
& \simeq \operatorname{hocolim}_{(n, m) \in \Delta^{o p} \times \Delta^{o p}} \bigvee_{\sigma \in S_{m}^{n}} A,
\end{aligned}
$$

where $S_{m}^{n}$ denotes the set $\left((F U)^{n} \Omega^{A} X\left(T_{1}\right)\right)_{m}$. 


\section{References}

[1] W. Chachólski, W.G. Dwyer, and M. Intermont. The A-complexity of a space. J. Lond. Math. Soc. (2) 65 (2002), no. 1, 204-222.

[2] E.D Farjoun. Cellular spaces, null spaces and homotopy localization. Lecture Notes in Math., 1622. Springer-Verlag, Berlin, 1996.

[3] P.S. Hirschhorn. Model categories and their localizations. Math. Surveys Monogr., 99. American Mathematical Society, Providence, RI, 2003.

[4] M. Sartwell. A P-local delooping machine. arXiv preprint arXiv:1510.08404, 2015.

[5] M. Sartwell. Detecting Mapping Spaces and Derived Equivalences of Algebraic Theories. Ph.D. thesis, State University of New York at Buffalo, 2016.

[6] S. Schwede. Stable homotopy of algebraic theories. Topology 40 (2001), no. 1, $1-41$.

[7] C.R. Stover. A van Kampen spectral sequence for higher homotopy groups. Topology 29 (1990), no. 1, 9-26.

Alyson Bittner alysonbi@buffalo.edu

Department of Mathematics, University at Buffalo, SUNY, Buffalo, NY 14260, USA 